\title{
PDGFRA-mutant syndrome
}

\author{
Riccardo Ricci ${ }^{1}$, Maurizio Martini ${ }^{1}$, Tonia Cenci ${ }^{1}$, Arnaldo Carbone ${ }^{1}$, Paola Lanza ${ }^{1}$,
} Alberto Biondi ${ }^{2}$, Guido Rindi ${ }^{1}$, Alessandra Cassano ${ }^{3}$, Alberto Larghi ${ }^{4}$, Roberto Persiani ${ }^{2}$ and Luigi M Larocca ${ }^{1}$

${ }^{1}$ Department of Pathology, Catholic University, Rome, Italy; ${ }^{2}$ Department of Surgery, Catholic University, Rome, Italy; ${ }^{3}$ Department of Oncology, Catholic University, Rome, Italy and ${ }^{4}$ Department of Digestive Endoscopy, Catholic University, Rome, Italy

\begin{abstract}
Germline PDGFRA mutations cause multiple heterogeneous gastrointestinal mesenchymal tumors. In its familial form this disease, which was formerly termed intestinal neurofibromatosis/neurofibromatosis $3 b$ (INF/NF3b), has been included among familial gastrointestinal stromal tumors (GISTs) because of its genotype, described when GIST was the only known PDGFRA-mutant gastrointestinal tumor. Shortly afterwards, however, inflammatory fibroid polyps also revealed PDGFRA mutations. Subsequently, gastrointestinal CD34+ 'fibrous tumors' of uncertain classification were described in a germline PDGFRA-mutant context. Our aim was to characterize the syndrome produced by germline PDGFRA mutations and establish diagnostic criteria and management strategies for this hitherto puzzling disease. We studied a kindred displaying multiple gastrointestinal mesenchymal tumors, comparing it with published families/individuals with possible analogous conditions. We identified a novel inherited PDGFRA mutation (P653L), constituting the third reported example of familial PDGFRA mutation. In adult mutants we detected inflammatory fibroid polyps, gastric GISTs and gastrointestinal fibrous tumors of uncertain nosology. We demonstrate that the syndrome formerly defined as INF/NF3b (exemplified by the family reported herein) is simplistically considered a form of familial GIST, because inflammatory fibroid polyps often prevail. Fibrous tumors appear variants of inflammatory fibroid polyps. 'INF/ NF3b' and 'familial GIST' are misleading terms which we propose changing to 'PDGFRA-mutant syndrome'. In this condition, unlike KIT-dependent familial GIST syndromes, if present, GISTs are stomach-restricted and diffuse Cajal cell hyperplasia is not observed. This restriction of GISTs to the stomach in PDGFRA-mutant syndrome: (i) focuses oncological concern on gastric masses, as inflammatory fibroid polyps are benign; (ii) supports a selective role of gastric environment for PDGFRA mutations to elicit GISTs, justifying the known predilection for stomach of sporadic PDGFRA-mutant GISTs. An awareness that inflammatory fibroid polyps, relatively common among gastrointestinal mesenchymal tumors, may be the prevailing tumor in PDGFRAmutant syndrome could eventually reveal an unsuspected prevalence of this condition.

Modern Pathology (2015) 28, 954-964; doi:10.1038/modpathol.2015.56; published online 15 May 2015
\end{abstract}

A dominantly inherited syndrome characterized by multiple gastrointestinal mesenchymal tumors of controversial nosology, formerly defined as intestinal neurofibromatosis/neurofibromatosis 3b (INF/ NF3b), has hitherto been described in two families. $^{1,2}$ It has been linked to a germline PDGFRA mutation $^{3}$ and, on account of this genotype, is currently classified among familial gastrointestinal stromal tumor (GIST) syndromes, despite controversial pathological evidence. ${ }^{4}$ However, GISTs are not the only gastrointestinal PDGFRA-mutant tumors; in

Correspondence: Dr R Ricci, MD, PhD, Department of Pathology, Catholic University, Largo A. Gemelli, 8, Rome I-00168, Italy. E-mail: riccardoricci@rm.unicatt.it

Received 16 January 2015; revised 20 March 2015; accepted 24 March 2015; published online 15 May 2015 fact, shortly after the INF/NF3b genotype was described, PDGFRA mutations were also found in inflammatory fibroid polyps. ${ }^{5-8}$ More recently, CD $34^{+}$fibrous tumors of uncertain classification (henceforth simply referred to as 'fibrous tumors') have been reported in a germline PDGFRA-mutated context, constituting another possible PDGFRAmutant tumor. ${ }^{9}$ Although GISTs and inflammatory fibroid polyps can sometimes be multiple and/or syndromic, ${ }^{9-14}$ germline PDGFRA mutations have rarely been described, with only two familial examples..$^{3,9,10,15}$ We had the opportunity of studying a kindred presenting heterogeneous gastrointestinal mesenchymal tumors bearing an unreported inherited PDGFRA mutation. This unusual scenario gave us the opportunity to attempt to fully characterize the disease formerly defined as INF/NF3b 
and establish adequate diagnostic criteria and management strategies. Concluding, we propose a new, objective denomination for this syndrome. Interestingly, the resulting redefinition of this disease could facilitate its diagnosis, possibly revealing a prevalence that is less rare than currently considered.

\section{Materials and methods}

\section{Patients}

A 67-year-old man (the proband, designated II-2 hereafter Figure 1) was referred to our hospital because of a palpable abdominal mass. After diagnosing multiple heterogeneous gastrointestinal tumors and a germline PDGFRA mutation, genetic screening and endoscopic surveillance where applicable were offered to his family members. The procedures followed were in accordance with the ethical standards of the local institutional committee on human experimentation and with the Helsinki declaration of 1975, as revised in 1983.

\section{Histology and Immunohistochemistry}

Sections from formalin-fixed, paraffin-embedded specimens were stained with hematoxylin/eosin. The antibodies used for immunohistochemistry were: CD117, S100 and vimentin (DAKO, Glostrup, Denmark), DOG1 (Spring Bioscience, Pleasanton, CA, USA), CD34 (Novocastra, Newcastle, UK) and PDGFRA (sc-338; Santa Cruz, Santa Cruz, CA, USA). Further details are reported in Supplementary Materials and Methods.

\section{Sequence Analysis in KIT and PDGFRA Genes}

Informed written consent was obtained from all tested individuals. DNA was obtained from paraffinembedded tissues and/or buccal swab samples. KIT (exons 9, 11, 13, and 17) and PDGFRA (exons 12 , 14, and 18) genes were amplified using the same primers and PCR conditions described elsewhere. ${ }^{16-18}$ Further details are reported in Supplementary Materials and Methods.

\section{Bioinformatics Analysis}

The P653L PDGFRA missense mutation was predicted with four different computational tools: 'SIFT', 19 'PolyPhen-2',20 'SNPs\&GO',21 and PROVEAN. ${ }^{22}$ Further details are reported in Supplementary Materials and Methods.

\section{Results}

\section{Family History}

The proband (individual II-2; Figure 1) is a man presenting at the age of 67 years with a palpable abdominal mass. CT-scan showed a 12-cm lesion originating from the gastric wall. Esophagogastroduodenoscopy additionally revealed a 2-cm polyp in the gastric antrum and subcentimetric duodenal pseudopolyps. The patient underwent partial gastrectomy, during which a right-colonic 8-cm polyp was also observed and resected. Pathology revealed two gastric GISTs (110 $\mathrm{mm}$ and $23 \mathrm{~mm}$ ), three gastric inflammatory fibroid polyps $(19 \mathrm{~mm}, 14 \mathrm{~mm}$, and $10 \mathrm{~mm}$ ), one right-colonic inflammatory fibroid



Figure 1 Pedigree of the kindred with a P653L-exon-14 PDGFRA mutation. Each generation is designated by a Roman numeral, and each family member by an Arabic numeral. Circles denote female family members, squares male family members, and slashes deceased family members. Below the symbols are indicated: first, age at death (i.e., 'd.47y') or at diagnosis of gastrointestinal tumor (ie, 'IFP 31y') or, in the absence of either events, current age; second, the PDGFRA genotype. 
polyp $(78 \mathrm{~mm})$, and three fibrous tumors $(18 \mathrm{~mm}$, $8 \mathrm{~mm}$, and $8 \mathrm{~mm}$ ) in the rim of duodenum. The latter extended from the submucosa to the muscularis propria in one case (which was fibrous/fatty), and to the subserosa in two cases, akin to those described by Carney and Stratakis. ${ }^{9}$ Moreover, a diffuse fibrous thickening was detected along the inner and outer aspects of gastric submucosa and in the submucosa of the duodenal rim. The patient began 3-year imatinib because of the size of the larger GIST and its rupturing during removal. ${ }^{23,24}$ Follow-up imaging (at 48 months) showed no recurrence. Family history revealed that the patient's father (I-2) had died of 'gastric cancer', without pathologic analysis; his halfsister (II-3) had had surgery for gastric, ileal and colonic tumors. After patient II-2 revealed a germline PDGFRA mutation, his relatives were offered genetic screening using buccal swab samples. In the presence of positivity, endoscopic evaluation was recommended. Thus, individuals III-3, III-4, and III-5 underwent esophagogastroduodenoscopy and ileocolonscopy. In individual III-3 (a 42-year-old woman), esophagogastroduodenoscopy revealed three gastric antral ( $7 \mathrm{~mm}, 10 \mathrm{~mm}$, and $15 \mathrm{~mm}$ ) and two $10-\mathrm{mm}$ duodenal polyps at the age 40 years. Endoscopic ultrasound showed the lesions to be confined to the superficial layers. PET/CT-scan was negative, and surveillance was adopted with the patient's consent. In individual III-4 (a 39-year-old woman) esophagogastroduodenoscopy revealed three antral polyps ( $4 \mathrm{~mm}, 4 \mathrm{~mm}$, and $25 \mathrm{~mm}$ ) at the age of 37 years. Endoscopic ultrasound-guided fineneedle tissue acquisition ${ }^{25}$ biopsies were consistent with inflammatory fibroid polyp in the two smaller lesions, but yielded no diagnostic samples from the larger one. PET/CT-scan was negative; however, the bigger lesion increased to $40 \mathrm{~mm}$ in 16 months. Partial gastrectomy was then performed, removing all tumors which pathologically proved inflammatory fibroid polyps; in addition, a 2-mm subserosal fibrous tumor was found. In individual III-5 (a 32year-old male), esophagogastroduodenoscopy revealed a $7-\mathrm{mm}$ antral polyp at the age of 31 years. Endoscopic ultrasound-guided fine-needle tissue acquisition biopsies were consistent with inflammatory fibroid polyp. Retrieved slides of tumors from individual II-3 (a 63-year-old woman; partial gastrectomy and right hemicolectomy in 1994, at the age of 43 years) revealed two gastric inflammatory fibroid polyps $(20 \mathrm{~mm}$ and $30 \mathrm{~mm})$, one $60-\mathrm{mm}$ ileocaecal valve inflammatory fibroid polyp, one colonic submucosal lipoma (12 mm), and four submucosal fibrous tumors (three ileal $(10 \mathrm{~mm}, 6 \mathrm{~mm}$, and $5 \mathrm{~mm}$ ), one duodenal (15 mm)). Moreover, three mesocolic fibrous/fatty tumors $(14 \mathrm{~mm}, 6 \mathrm{~mm}$, and $4 \mathrm{~mm}$ ) were found. At the time of operation, these findings were diagnosed as 'consistent with INF', but no genetic counseling followed. The patient is alive and asymptomatic; instrumental follow-up was refused.

\section{Histology and Immunohistochemistry}

For practical purposes, in this 'Results' section, both the presence of numerous eosinophils ( $>10 / \mathrm{HPF}$ ) and lack of evidence of tumor involvement deeper than submucosa are arbitrarily considered necessary features for diagnosing classical inflammatory fibroid polyps; non GISTs lacking one or both of these features showed many similarities to the 'fibrous tumors' described by Carney and Stratakis, ${ }^{9}$ and are referred to as such. The overlaps and possible relationships between inflammatory fibroid polyps and fibrous tumors will be extensively treated in the 'Discussion' section.

Classical inflammatory fibroid polyps, found in individuals II-2, II-3, III-4, and III-5, involved mucosal lamina propria and/or submucosa, and were typically composed of an admixture of fibroblast-like mesenchymal cells and inflammatory cells, including numerous eosinophils (ranging between 15 and 160 per $\mathrm{HPF}$ ), and mast cells, in a myxoid collagenous matrix, often organized in an onion-skin pattern around blood vessels and displaying a short-fascicular growth pattern (Figure 2a and b). Gastric inflammatory fibroid polyps in individual II-2 sometimes stemmed from a fibrous thickening of submucosa along its inner limit (Figure 2c); a similar fibrous thickening lined the gastric submucosal outer limit and involved the submucosa of the duodenal rim, superficially penetrating muscularis propria; both these fibrous thickenings were composed of tissue similar to fibrous tumors (described in detail below). At immunohistochemistry, inflammatory fibroid polyps proved vimentin+ (not shown), and CD34 ${ }^{+}$(intensely and diffusely in all gastric cases (Figure 2d) and focally in one out of two colonic examples); PDGFRA staining was variable (Figure 2e and f); CD117, S100, and DOG 1 were negative (not shown).

Fibrous tumors, found in individuals II-2, II-3, and III-4, variably involved mucosal lamina propria, submucosa, muscularis propria, and/or subserosa. They were unencapsulated; their borders were at times circumscribed, even sharply delineated, at times irregular and infiltrative (this was the rule whenever fibrous tumors involved muscularis propria). Fibrous tumors were composed of polygonalto-spindle cells with little acidophilic cytoplasm and small, slightly irregular nuclei, with fine evenly dispersed chromatin, and small nucleoli, in a heavily collagenized background; however, an onion-skin pattern around blood vessels and/or eosinophils (ranging between 0-15 per HPF) could be observed, although in a minority of cases; mast cells were scattered throughout (Figure 3a and b). In individual II-2, fibrous thickenings with histologic features similar to fibrous tumors lined the gastric submucosa along its inner and outer limits (Figure 3c) and involved the submucosa of the duodenal rim. In some fibrous tumors from individuals II-2 and II-3 intermingled adipocytes were found, conferring a fibrous/ fatty appearance (Figure $3 \mathrm{~d}$ shows a duodenal example from individual II-2). At immunohistochemistry, fibrous 

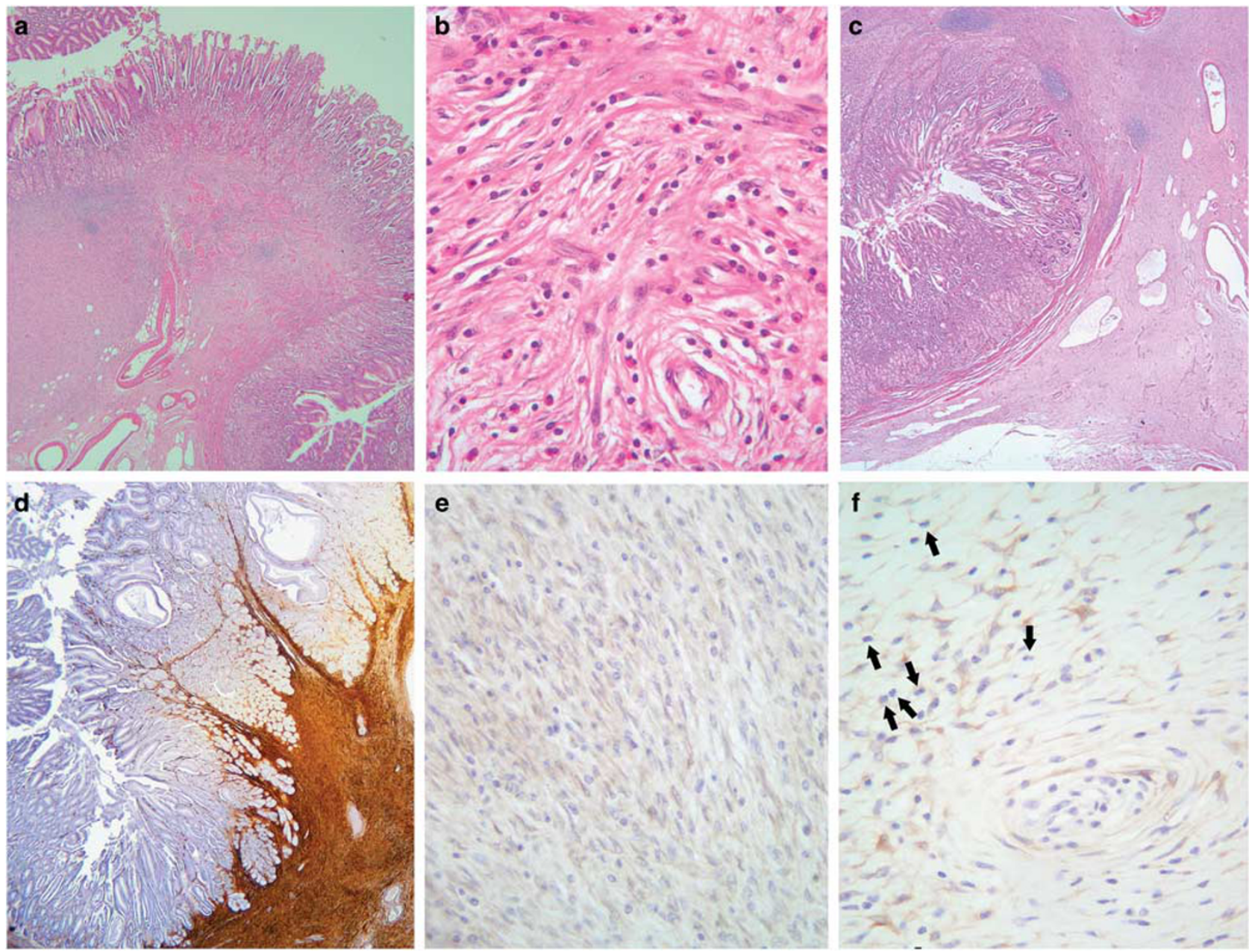

Figure 2 Representative examples of histological and immunohistochemical images of inflammatory fibroid polyp, and submucosal fibrous thickening accompanying it in individual II-2. A gastric inflammatory fibroid polyp at panoramic view (a, H\&E) and at high-power detail (b, H\&E). In individual II-2, gastric inflammatory fibroid polyps (c top, H\&E) stemmed from a fibrous thickening of submucosa along its inner limit (c bottom, H\&E). Strong and diffuse immunohistochemical CD34-positivity is typical of gastric inflammatory fibroid polyps (d); PDGFRA immunohistochemical positivity can either be very slight (e) or more intense (f; notice the well developed onion-skin pattern and the presence of eosinophils, arrows highlight their bilobed nuclei, unequivocally qualifying the lesion as a classical inflammatory fibroid polyp).

tumors proved $\mathrm{CD}_{117^{-}}$, CD34 intensely and diffusely ${ }^{+}$in all cases but one, PDGFRA ${ }^{+}$in most cases (Figure 3e, $\mathrm{f}$ and g, respectively), vimentin ${ }^{+}, \mathrm{DOG1}^{-}$, and $\mathrm{S} 100^{-}$(not shown). Gastric sumbucosal fibrous thickening found in individual II-2 displayed analogous immunohistochemical features (Figure $3 \mathrm{~h}$ and i show CD34 and PDGFRA immunostaining, respectively).

Gastric GISTs of individual II-2 measured $110 \mathrm{~mm}$ and $23 \mathrm{~mm}$ and showed an epithelioid and mixed phenotype, (Figure $4 \mathrm{a}$ and $\mathrm{b}$ ) and 2 and 6 mitoses per 50 $\mathrm{HPF}$, respectively; the first was classified as high-risk because rupturing occurred during removal. ${ }^{24}$ At immunohistochemistry, GISTs proved heterogeneously CD117 + (Figure 4c) and $\mathrm{DOG}^{+}$(Figure 4d), and PDGFRA ${ }^{+}$ (Figure 4e); CD34 and vimentin stainings were also positive, whereas S100 was negative (not shown).

Colonic lipoma, found in individual II-3, was a circumscribed but unencapsulated mass composed of mature fat cells (not shown).
Table 1 summarizes morphology and immunohistochemical features of the resected tumors.

\section{Sequence Analysis in KIT and PDGFRA Genes}

A germline mutation (CC $\rightarrow$ TT at 1957-1958) determining a Leu for pro-substitution at 653 (P653L) in PDGFRA gene (Figure 5) was found in individuals II-2, II-3, III-3, III-4, III-5, IV-1, IV-2, IV-4, IV-6, and IV-7; individuals III-1, III-2, IV-3, and IV-5 were PDGFRA-wild type (Figure 1), as were the wives of patients II-2 and III-5 (controls). KIT was wild type in all tumoral (including GISTs) and nontumoral tissue samples from all individuals.

\section{Bioinformatics Analysis}

P653L PDGFRA was predicted with four different computational tools; all indicated protein damage. 
In detail: SIFT score (range $0=$ damaging to $1=$ tolerated), 0.03; PolyPhen-2 score (range $0=$ benign to 1 = damaging), 0.998; SNPs\&GO ('disease-related' or 'neutral', with a reliability index ranging from 0 to 10), 'disease'5; PROVEAN score $(<-2.5=$ 'deleterious'; $>-2.5=$ 'neutral'), $-5.798 .^{19-22}$

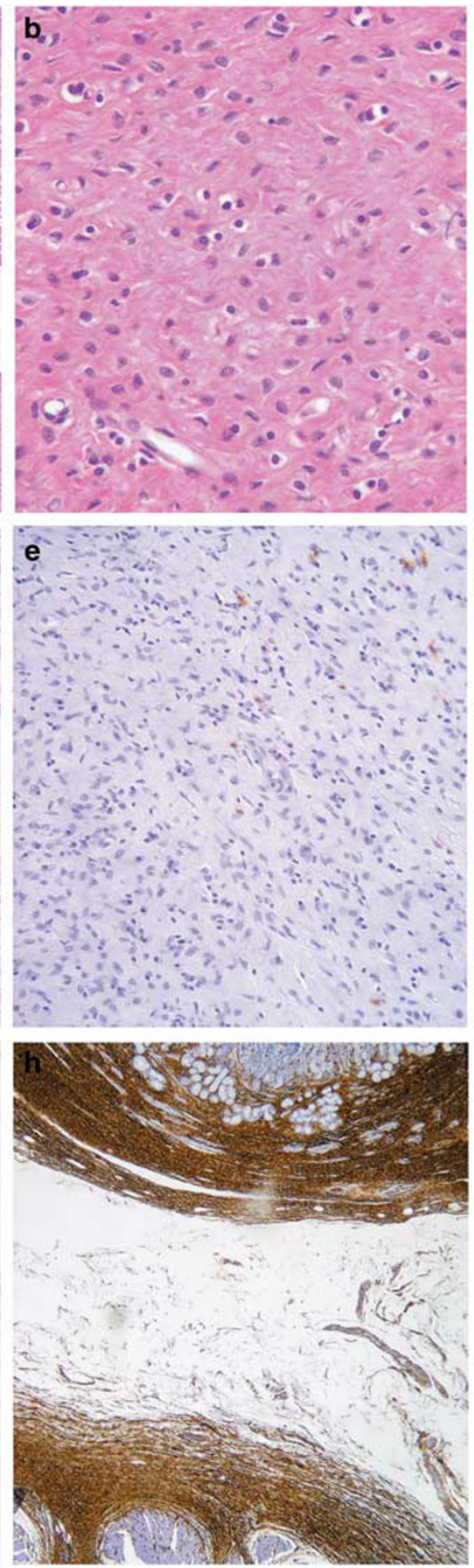
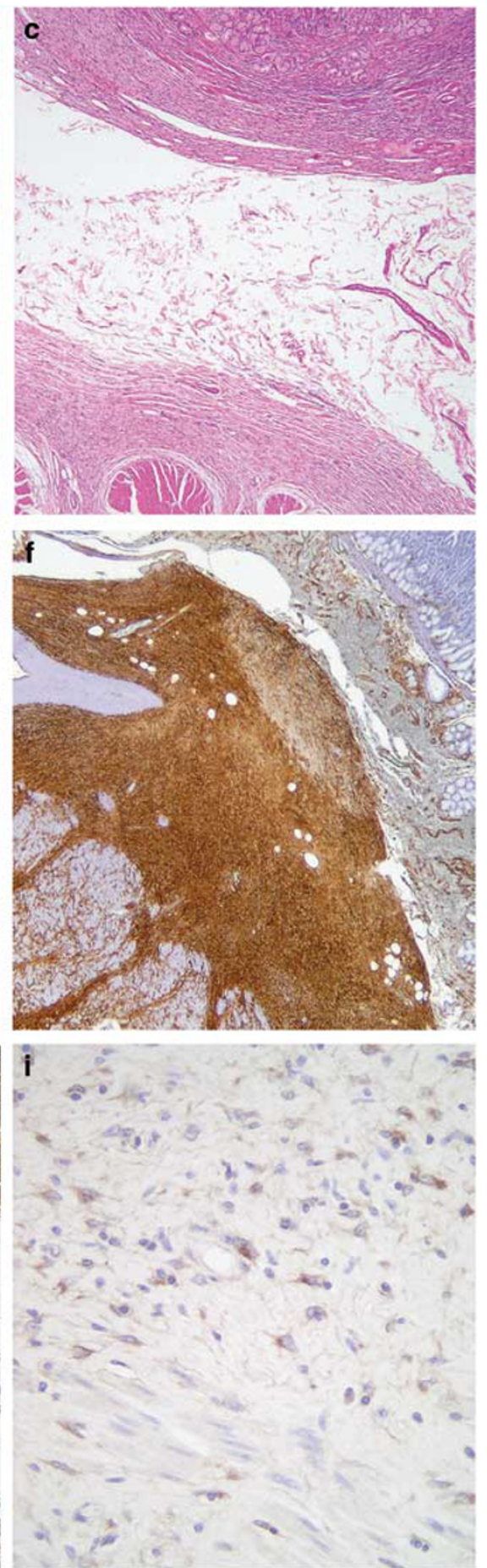

Figure 3 Representative examples of histological and immunohistochemical images of fibrous tumors, and gastric submucosal fibrous thickenings found in individual II-2. Fibrous tumor at panoramic view (a, H\&E) and at high-power detail (b, H\&E). In individual II-2, a fibrous thickening resembling fibrous tumors was found along the inner and outer aspects of gastric submucosa (c, H\&E). Duodenal fibrous/fatty tumor from individual II-2 at panoramic view (d, H\&E). Fibrous tumor typically showed immunohistochemical negativity for CD117 (e, scattered mast cells stain positively) and, with the exception of one case, diffuse and intense CD34-positivity (f). PDGFRA resulted often positive (g; compare with the negativity of smooth-muscle at bottom left). Similar to fibrous tumor, gastric submucosal fibrous thickenings found in individual II-2 were intensely CD34-positive (h) and PDGFRA-positive (i; again, adjacent non-tumoral smooth-muscle tissue at the bottom was negative). 

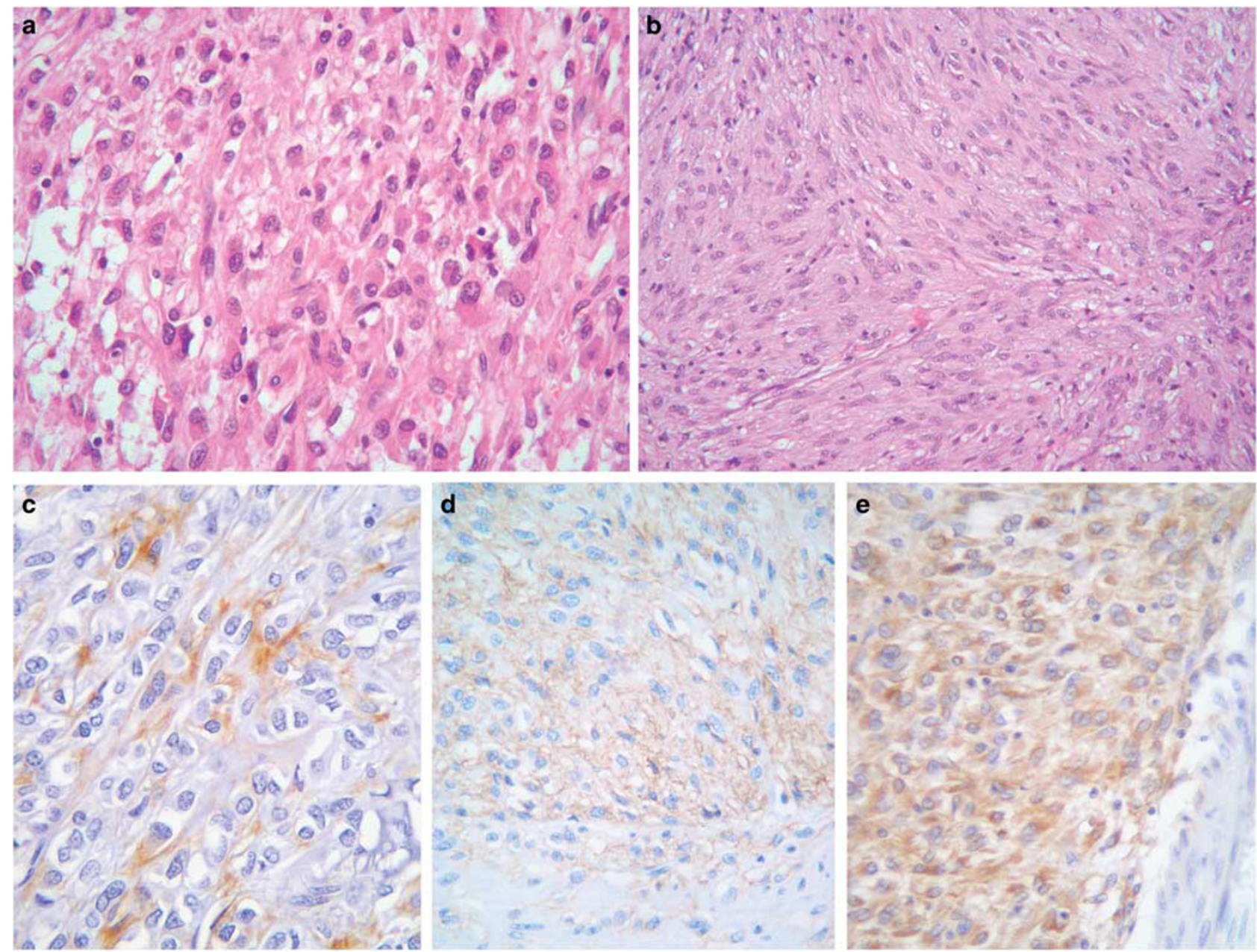

Figure 4 Representative examples of histological and immunohistochemical images of GIST. Both gastric GISTs, found in individual II-2, showed an epithelioid morphology (a, larger GIST, H\&E); in the smaller GIST spindle-cell areas were also detected (b, H\&E). CD117 immunostain revealed a heterogeneous positivity (c) as did DOG1 (d; non-tumoral smooth-muscle tissue at the bottom was negative); PDGFRA staining resulted positive (e; adjacent non-tumoral smooth-muscle tissue at bottom right showed absent background signal).

\section{Discussion}

We herein report a kindred displaying inflammatory fibroid polyps, GISTs, and fibrous tumors, bearing an inherited P653L mutation in PDGFRA exon-14.

PDGFRA mutations are reported in about $74 \%$ and $55 \%$ of gastric and small-intestinal inflammatory fibroid polyps, respectively, ${ }^{7,8}$ and in $6-7 \%$ of GISTs. $^{5,6}$ PDGFRA exon-14 mutations occur in $<1 \%$ of GISTs $^{26}$ and, to the best of our knowledge, have very rarely been reported in inflammatory fibroid polyps. ${ }^{27}$

P653L PDGFRA mutation is hitherto unreported. ${ }^{28}$ Highly conserved amino acid alignment of tyrosine kinase I PDGFRA and KIT domains shows correspondence between codon 653 of PDGFRA and codon 649 of KIT (exon 13), whose substitutions have also never been reported. ${ }^{28}$ P653L PDGFRA can affect protein function, as predicted by four different computational tools. ${ }^{19-22}$
PDGFRA immunoreactivity, variable in inflammatory fibroid polyps and fibrous tumors, and evident in GISTs, was faintly ${ }^{+} /$negative in non-tumoral tissue (Figures 2e and f, $3 g$ and i, and 4e). Although PDGFRA immunohistochemistry can be problematic, ${ }^{29}$ these consistently observed differences in PDGFRA immunoreactivity probably conceal additional events of PDGFRA tumorigenesis in relation to the germline PDGFRA mutation in background.

The fibrous tumors we described strongly resemble those found in another germline PDGFRA-mutant case and, likewise, did not metastasize. ${ }^{9}$ In the same case, Carney and Stratakis ${ }^{9}$ found lipomatous tumors, as we did in the colon of individual II-3. A tumor similar to fibrous tumors has also been described in the ileum of a 82-year-old male; in this case the finding of a double mutation in PDGFRA exon-14 only in a fraction of tumor cells invalidates the possibility of a germline mutation. ${ }^{30}$ Fibrous tumors are morphologically and immuno- 
Table 1 Morphologic and immunohistochemical features of gastrointestinal tumors resected in the reported kindred

\begin{tabular}{|c|c|c|c|c|c|c|c|c|c|c|c|c|c|c|c|c|c|c|c|c|c|c|}
\hline \multirow{2}{*}{$\begin{array}{l}\text { Tumor } \\
\text { type }\end{array}$} & \multirow{2}{*}{ Individual } & \multirow{2}{*}{ Site } & \multicolumn{4}{|c|}{ Layer involvement } & \multirow{2}{*}{ Size (mm) } & \multicolumn{2}{|c|}{ Shape } & \multicolumn{5}{|c|}{ Architecture } & \multicolumn{2}{|c|}{ Leukocytes } & \multicolumn{6}{|c|}{$I H C$} \\
\hline & & & $L P^{\mathrm{a}}$ & $S M^{\mathrm{b}}$ & $M P^{c}$ & $S S^{\mathrm{d}}$ & & $\begin{array}{l}\text { Polipoid } \\
\text { shape }\end{array}$ & $\begin{array}{l}\text { Sharp } \\
\text { boders }\end{array}$ & $\begin{array}{l}\text { Blood } \\
\text { vessels }\end{array}$ & $\begin{array}{l}\text { Onion-skin } \\
\text { pattern }\end{array}$ & $\begin{array}{l}\text { Short } \\
\text { fasicles }\end{array}$ & Edema & $\begin{array}{l}\text { Collageni- } \\
\text { zation }\end{array}$ & $\begin{array}{c}\text { Eosinophils/ } \\
\text { HPF }^{\mathrm{e}}\end{array}$ & $\begin{array}{c}\text { Mononuclear } \\
\text { cells }\end{array}$ & $C D 34$ & PDGFRA & CD117 & DOG1 & $S 100$ & Vimentin \\
\hline \multicolumn{23}{|c|}{ Inflammatory fibroid polyp } \\
\hline 护 & II-2 & Stomach & Yes & Yes & No & No & 19 & Yes & No & $+++^{\mathrm{f}}$ & +++ & Yes & $\begin{array}{c}+ \text { Sup } \\
+++ \text { Deepg }\end{array}$ & $\begin{array}{l}+ \text { Sup } \\
+++ \text { Deep }\end{array}$ & 37 & +++ & Pos $^{\text {h }}$ & + & $\mathrm{Neg}$ & $\mathrm{Neg}$ & Neg & Pos \\
\hline & II-2 & Stomach & Yes & Yes & No & No & 14 & Yes & No & +++ & +++ & Yes & ++ & $\begin{array}{l}+ \text { Sup } \\
+++ \text { Deep }\end{array}$ & 62 & +++ & Pos & + & $\mathrm{Neg}$ & $\mathrm{Neg}$ & Neg & Pos \\
\hline & II-2 & Stomach & Yes & Yes & No & No & 10 & Yes & No & +++ & +++ & Yes & ++ & ++ & 63 & +++ & Pos & + & $\mathrm{Neg}$ & $\mathrm{Neg}$ & Neg & Pos \\
\hline & III-2 & Right colon & Yes & Yes & $N A^{i}$ & $N A^{i}$ & 78 & Yes & No & +++ & +++ & Focal & ++ & ++ & 20 & +++ & $\mathrm{Neg}$ & +++ & $\mathrm{Neg}$ & $\mathrm{Neg}$ & Neg & Pos \\
\hline & II-3 & Stomach & Yes & Yes & No & No & 30 & Yes & No & +++ & +++ & Focal & ++ & ++ & 104 & +++ & Pos & + & Neg & Neg & Neg & Pos \\
\hline & II-3 & Stomach & Yes & Yes & No & No & 20 & Yes & No & +++ & +++ & Yes & +++ & + & 28 & +++ & Pos & + & Neg & Neg & Neg & Pos \\
\hline & II-3 & $\begin{array}{l}\text { Ileocaecal } \\
\text { valve }\end{array}$ & Yes & Yes & No & No & 60 & Yes & No & t++ & t++ & No & +++ & ++ & 15 & ++ & Pos/neg & $\begin{array}{l}\text { T } \\
++\end{array}$ & Neg & Neg & Neg & Pos \\
\hline & III-4 & Stomach & Yes & Yes & No & No & 42 & No & $\begin{array}{l}\text { No sup } \\
\text { Yes deep }\end{array}$ & ++ & ++ & Yes & + & +++ & 31 & ++ & Pos & ++ & Neg & $\mathrm{Neg}$ & Neg & Pos \\
\hline & III-4 & Stomach & Yes & Yes & No & No & 18 & Yes & $\begin{array}{l}\text { No sup } \\
\text { Yes deep }\end{array}$ & $\begin{array}{l}\text { +++ Sup } \\
\text { + Deep }\end{array}$ & $\begin{array}{l}+++\mathrm{LP} \\
-\mathrm{SM}\end{array}$ & $\begin{array}{l}\text { Yes sup } \\
\text { No deep }\end{array}$ & ++ & ++ & $\begin{array}{l}112 \text { Sup } \\
22 \text { Deep }\end{array}$ & $\begin{array}{l}\text { +++ Sup, } \\
\text { ++ Deep }\end{array}$ & Pos & +++ & Neg & $\mathrm{Neg}$ & $\mathrm{Neg}$ & Pos \\
\hline & III-4 & Stomach & Yes & Yes & No & No & 13 & Yes & $\begin{array}{l}\text { No sup } \\
\text { Yes deep }\end{array}$ & +++ & +++ & No & ++ & ++ & 160 & +++ & Pos & + & $\mathrm{Neg}$ & Neg & Neg & Pos \\
\hline \multicolumn{23}{|c|}{ Fibrous tumor } \\
\hline & III-2 & Duodenum & No & Yes & Yes & Yes & 18 & No & No & + & - & No & - & +++ & 11 & + & Pos & - & $\mathrm{Neg}$ & $\mathrm{Neg}$ & Neg & Pos \\
\hline & III-2 & Duodenum & No & Yes & Yes & Yes & 8 & No & No & + & - & No & + & +++ & 0 & ++ & Pos & - & $\mathrm{Neg}$ & Neg & Neg & Pos \\
\hline & II-3 & Duodenum & No & Yes & No & No & 15 & No & No & + & - & No & - & +++ & 0 & + & Pos & + & $\mathrm{Neg}$ & $\mathrm{Neg}$ & Neg & Pos \\
\hline & III-3 & Ileum & Yes & Yes & No & No & 10 & No & $\begin{array}{l}\text { No sup } \\
\text { Yes deep }\end{array}$ & ++ & +++ & No & - & +++ & 0 & + & Pos & +++ & Neg & Neg & Neg & Pos \\
\hline & II-3 & Ileum & No & Yes & No & No & 6 & No & Yes & + & + & No & - & +++ & 0 & + & Neg & ++ & $\mathrm{Neg}$ & $\mathrm{Neg}$ & $\mathrm{Neg}$ & Pos \\
\hline & II-3 & Ileum & Yes & Yes & No & No & 5 & No & $\begin{array}{l}\text { No sup } \\
\text { Yes deep }\end{array}$ & ++ & ++ & No & + & +++ & 7 & ++ & Pos & +++ & Neg & Neg & Neg & Pos \\
\hline & III-4 & Stomach & No & No & Yes & Yes & 2 & No & No & + & - & No & - & +++ & 0 & ++ & Pos & +++ & Neg & Neg & Neg & Pos \\
\hline \multicolumn{23}{|c|}{ Fibrous/fatty tumor } \\
\hline & II-2 & Duodenum & No & Yes & Yes & No & 8 & No & No & - & - & No & + & +++ & 15 & ++ & Pos & - & $\mathrm{Neg}$ & $\mathrm{Neg}$ & $\mathrm{Neg}$ & Pos \\
\hline & III-3 & Mesocolon & No & No & No & Yes & 14 & No & No & + & - & No & - & +++ & 0 & ++ & Pos & ++ & Neg & Neg & Neg & Pos \\
\hline & II-3 & Mesocolon & No & No & No & Yes & 6 & No & No & + & - & No & - & +++ & 0 & + & Pos & ++ & $\mathrm{Neg}$ & $\mathrm{Neg}$ & Neg & Pos \\
\hline & II-3 & Mesocolon & No & No & No & Yes & 4 & No & No & + & - & No & - & +++ & 2 & + & Pos & ++ & $\mathrm{Neg}$ & $\mathrm{Neg}$ & Neg & Pos \\
\hline \multicolumn{23}{|c|}{ Diffuse submucosal fibrous thickening } \\
\hline & II-2 & Stomach & No & Yes & Yes & No & $N A^{j}$ & No & No & + & - & No & ++ & $++/+++$ & 6 & +/++ & Pos & - & Neg & $\mathrm{Neg}$ & Neg & Pos \\
\hline Lipoma & II-3 & Right colon & No & Yes & No & No & 12 & No & Yes & + & - & No & - & - & 0 & - & $\mathrm{ND}^{\mathrm{k}}$ & $\mathrm{ND}$ & ND & ND & ND & ND \\
\hline \multirow[t]{2}{*}{ GIST } & & & & & & & & & & & & & & & & & & & & & & \\
\hline & $\begin{array}{l}\text { II-2 } \\
\text { II-2 }\end{array}$ & $\begin{array}{l}\text { Stomach } \\
\text { Stomach }\end{array}$ & $\begin{array}{l}\text { No } \\
\text { No }\end{array}$ & $\begin{array}{l}\text { No } \\
\text { No }\end{array}$ & $\begin{array}{l}\text { Yes } \\
\text { Yes }\end{array}$ & $\begin{array}{l}\text { Yes } \\
\text { Yes }\end{array}$ & $\begin{array}{r}23 \\
110\end{array}$ & $\begin{array}{l}\text { No } \\
\text { No }\end{array}$ & $\begin{array}{l}\text { Mostly } \\
\text { Yes }\end{array}$ & $\begin{array}{l}+ \\
+\end{array}$ & $\bar{z}_{-}$ & $\begin{array}{l}\text { No } \\
\text { No }\end{array}$ & - & $\bar{z}_{-}$ & $\begin{array}{l}0 \\
0\end{array}$ & $\begin{array}{c}+ \\
++\end{array}$ & $\begin{array}{l}\text { Pos } \\
\text { Partly pos }\end{array}$ & $\begin{array}{l}+++ \\
+++\end{array}$ & $\begin{array}{l}\text { Partly pos } \\
\text { Neg }\end{array}$ & $\begin{array}{l}\text { Neg } \\
\text { Partly pos }\end{array}$ & $\begin{array}{l}\mathrm{Neg} \\
\mathrm{Neg}\end{array}$ & $\begin{array}{l}\text { Pos } \\
\text { Pos }\end{array}$ \\
\hline
\end{tabular}

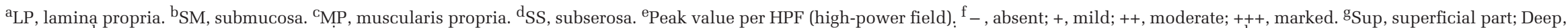
deep part. ${ }^{h}$ Pos, positive; Neg, negative. ${ }^{i}$ NA, not applicable because resection specimen did not include MP and SS. ${ }^{j}$ NA because the lesion was diffuse. ${ }^{k}$ ND, not determined. 




Figure 5 Gene mutation from non-tumoral tissue in a P653L PDGFRA-mutation carrier of the described kindred. Partial nucleotide sequence of exon-14 of PDGFRA gene revealed a mutation ( $\mathrm{CC} \rightarrow \mathrm{TT}$ at 1957-1958) determining a Leu for Prosubstitution at 653 (P653L) in both tumoral and non-tumoral tissue (a control lymph node from individual II-2 is shown; analogous results were obtained from all studied tissues from $\mathrm{P} 653 \mathrm{~L}$ PDGFRA-mutation carriers of the investigated kindred, either non-tumoral or tumoral, irrespective of histotype).

phenotypically $\left(\mathrm{CD} 117^{-}, \mathrm{DOG}^{-}\right)$readily distinguishable from GISTs. Conversely, they share both morphologic and immunophenotypic features with inflammatory fibroid polyps, which can present with different pathological features possibly related to anatomic site and/or evolutional changes. In fact, like fibrous tumors, inflammatory fibroid polyps may feature a prominent hyalinization, lack of a polypoid shape and, especially in intestinal cases, involvement of visceral layers deeper than submucosa, absence of eosinophils and lack of an onion-skin pattern, and CD34 staining. ${ }^{31-35}$ In particular, fibrous tumors resemble the sclerotic stage of inflammatory fibroid polyps described by Kim and Kim. ${ }^{33}$ These findings, together with the common PDGFRA-mutant status, in our opinion, well support the inclusion of fibrous tumors in the inflammatory fibroid polyp family. In this perspective, it is significant that in individual II-2 inflammatory fibroid polyps stemmed from a diffuse fibrous thickening of submucosa resembling the fibrous tumors.

Sporadic PDGFRA-mutant GISTs are known to be linked to a gastric location. ${ }^{5,26}$ Interestingly, this happens even in the case of germline PDGFRA mutations, a context where other PDGFRA-driven tumors (inflammatory fibroid polyps and fibrous tumors if considered separately) arise also in the bowel (as shown here and in refs. 9 and 10). This event, which to date has never been highlighted, demonstrates that the stomach has a relatively selective role for PDGFRA mutations to elicit GISTs, possibly owing to differences in gene expression secondary to gastric environment. Similarly, anatomic site influences gene expression in KIT-driven GISTs, even in germline KIT mutations. ${ }^{36}$ Furthermore, stomach could host a peculiar cell-type that, upon PDGFRA mutation, is prone to progress to GIST. This hypothesis is also supported by the frequent detection of diffuse interstitial Cajal cell hyperplasia in germline KIT mutations ${ }^{5}$ but not in germline PDGFRA mutations; in fact, not only did we fail to find diffuse interstitial Cajal cell hyperplasia in any of the mutant individuals of the kindred herein reported, but diffuse interstitial Cajal cell hyperplasia has never been described in germline PDGFRA-mutant contexts. , $^{3,10,15}$ Thus, the common statement that GISTs derive from interstitial Cajal cells may not be valid for PDGFRA-mutant GISTs or, in the histogenesis of this GIST subtype, there may be an early stage that passes through a cell-type devoid of usual features of interstitial Cajal cells detectable by histology and immunohistochemistry.

The frequent association between inflammatory fibroid polyps and GISTs in germline PDGFRAmutant contexts could apparently renew the question of a possible pathogenetic relationship between these two tumors. Conflicting with such a relationship is the lack of evidence of hybrid tumors, i.e. residual inflammatory fibroid polyps beside overt PDGFRA-mutant GISTs or inflammatory fibroid polyps hosting micro GISTs; the literature reports only rare adjacent but well distinguished GISTs and inflammatory fibroid polyps. ${ }^{7}$ Moreover, independent small GISTs $(\leq 3 \mathrm{~mm})$ have been described in the stomach of a germline PDGFRA-mutant individual, together with overt, larger tumors of this kind: ${ }^{9}$ this finding suggests that PDGFRA-driven GISTs display GIST features from their onset.

Several favorable prognostic clues are present in our kindred: inflammatory fibroid polyps are benign, ${ }^{7,8}$ as are fibrous tumors if considered separately; ${ }^{9,30}$ PDGFRA-driven GISTs are relatively indolent, ${ }^{5}$ especially those with PDGFRA exon-14 mutations; ${ }^{26}$ individual II-2 is free-of-disease 48 months after GIST rupture at surgery (with 3-year imatinib therapy); the detection of the hitherto unreported germline P653L PDGFRA mutation in an apparently unrelated individual (whose family was not studied) from a village $100 \mathrm{~km}$ away from individual II-2's dwelling, displaying inflammatory fibroid polyps and a GIST (RR, unpublished data), suggests a possible ancient origin of $\mathrm{P} 653 \mathrm{~L}$ and its compatibility with life and reproduction.

Fibrous tumors and/or GISTs associated with germline PDGFRA mutations have rarely been described. ${ }^{3,9,10,15}$ In the family reported by Chompret et $a 1^{10}$ a D846Y PDGFRA exon 18 mutation cosegregated with well documented GISTs (CD117 ${ }^{+}$, CD34 ${ }^{+}$). An individual bearing a germline V561D PDGFRA exon 12 mutation (family was unavailable for studies) displayed multiple gastric GISTs, and gastrointestinal fibrous tumors and lipomas. ${ }^{9,15}$ Lastly, the kindred reported by de Raedt et $a l^{3}$ is an example of the syndrome formerly defined as INF/NF3b, currently considered a form of familial GIST. ${ }^{4}$

Although the term 'INF' has been used with various meanings, to the best of our knowledge it has defined a well-characterized hereditary syndrome (INF/NF3b) in two families only. ${ }^{1,2}$ This dominantly inherited condition, manifesting gastrointestinal tumors formerly diagnosed as neurofibromata, without other features of either type 1 or 2 
neurofibromatosis, and displaying intestinal obstruction/bleeding owing to intussusception, without malignancies, has been linked to an inherited PDGFRA mutation. In fact, a Y555C PDGFRA exon 12 germline mutation was found in three sisters from an INF/NF3b kindred, and the 'neurofibromata' found in this family were re-considered KIT-negative GISTs, ${ }^{3}$ leading to including INF/NF3b among familial GIST syndromes. ${ }^{4}$ However, the only INF/ NF3b-associated 'neurofibromata' studied by immunohistochemistry proved S100', CD117' ${ }^{-}$, and $\mathrm{CD}^{-} 4^{-}$; moreover, DOG1 and PKC-theta were also negative by in situ hybridization/western blot, and KIT and NF1 proved wild type. ${ }^{3}$ Thus, these tumors showed neither neurofibroma features, nor GIST descriptors other than a PDGFRA mutation, a genetic feature demonstrated shortly afterwards also in inflammatory fibroid polyps ${ }^{7,8}$ and subsequently in fibrous tumors. ${ }^{9,15}$ Conversely, their reported features (intraluminal/polypoid appearance, white solid aspect, collagenous background, mast cells and lack of mitoses) and invariably benign behavior ${ }^{2,3}$ could well fall within the spectrum of inflammatory fibroid polyps (noticeably often $\mathrm{CD}^{-} 4^{-}$in the small intestine $^{8}$ ) and/or fibrous tumors, if considered separately. Classifying INF/NF3b as a form of familial GIST has at times been seen as an excessively simplistic approach. ${ }^{37}$ Incidentally, if the tumors studied by de Raedt et $a l^{3}$ are not GISTs, we herein report the second PDGFRA-driven familial condition presenting GISTs after that described by Chompret et al. ${ }^{10}$ Of note is the GIST-bearing germline PDGFRA-mutant case reported by Pasini et al ${ }^{15}$ and Carney and Stratakis ${ }^{9}$ that has been referred to as an example of PDGFRA-dependent familial GIST ${ }^{38}$ despite the lack of evidence of both gastrointestinal tumors and genetic investigations in patient's relatives.

Interestingly, three families displaying multiple inflammatory fibroid polyps have been described without mutational data, ${ }^{11-14}$ and 'neurofibromata' or even neurofibromatosis had previously been pathologically diagnosed in gastrointestinal tumors from patients later recognized as members of kindreds with familial multiple inflammatory fibroid polyps $^{11}$ or found to bear a germline PDGFRA mutation. ${ }^{9}$ These events support the overlap between these conditions and the disease formerly called INF/NF3b.

Curiously, a 'neurofibroma' from the INF/NF3b family published by Heimann et $a l^{2}$ showed intratumoral adipocytes, suggesting a possible relationship with the intestinal fibrous/fatty tumors and lipomas described by Carney and Stratakis $^{9}$ in a germline mutant PDGFRA exon 12 context and with the fibrous/fatty tumors and the lipoma we observed in individuals II-2 and II-3. Of note is the association between gastrointestinal lipomas and GISTs that has been reported also in two cases without both genotyping and evidence of familial transmission: significantly, GISTs always featured a gastric location and an epithelioid morphology, ${ }^{39,40}$ both suggestive of a possible PDGFRA-driven pathogenesis.

Finally, large hands were present in two PDGFRAmutant kindreds: one with clear-cut evidence of gastric GISTs but no mention of a 'neurofibromatous' phenotype; ${ }^{10}$ the other constituting one of the aforementioned families with INF/NF3b and, as previously stated, without convincing evidence of GISTs. ${ }^{3}$ Large hands were not observed in the kindred reported in our present study.

We therefore propose considering our kindred, those reported by Chompret et $a l^{10}$ and De Raedt et $a l,{ }^{3}$ and the case described by Pasini et $a l^{15}$ and Carney and Stratakis ${ }^{9}$ as different manifestations of a common phenotypic spectrum related to germline PDGFRA mutations, with autosomal dominant transmission, whose possible manifestations include gastrointestinal mesenchymal tumors (inflammatory fibroid polyps, including fibrous tumors, fatty tumors and gastric GISTs) and large hands, with an overall benign clinical course, coinciding with the disease formerly defined as INF/NF3b. ${ }^{1-3}$ Possible differences in phenotype probably depend on the specific PDGFRA mutation involved. Hereditary inflammatory fibroid polyp syndromes ${ }^{11-14}$ and cases featuring both gastric GISTs and gastrointestinal lipomas ${ }^{39,40}$ also possibly belong to this group; an assessment of their PDGFRA status could be confirmatory. The unprecedented awareness that inflammatory fibroid polyp, a relatively frequent lesion among gastrointestinal mesenchymal tumors, ${ }^{41}$ and gastrointestinal lipomas are part of the spectrum of 'INF/NF3b', at least the former often accounting for the majority of tumors found in affected individuals, could increase the detection rate of 'INF/NF3b', possibly ultimately showing that this syndrome is not as rare as was formerly believed.

As 'INF/NF3b' and 'familial GIST' appear to be misleading terms for defining the disease investigated, we therefore propose adopting the term 'PDGFRA-mutant syndrome'.

Clinically speaking, endoscopy combined with endoscopic ultrasound and/or entero CT appear to be appropriate techniques for studying PDGFRAmutant syndrome patients. Potential harmful events can be of a 'mechanical' or oncological nature. The former usually consist of bowel obstruction/ intussusception. ${ }^{2,3,9,11-15}$ Oncologic risk appears to be mainly restricted to gastric tumors, as the stomach proved to be selective for PDGFRA mutations to progress to GIST, and GIST appears to be the only tumor potentially malignant in PDGFRA-mutant syndrome. This indicates that although histological assessment of tumors (i.e., using endoscopic ultrasound-fine-needle tissue acquisition ${ }^{25}$ ) is always recommended, it is particularly warranted in gastric tumors. Whatever the type of risk, a surgical option should be considered for every tumor in PDGFRAmutant syndrome; this must apply to tumors diagnosed as GIST following current guidelines, ${ }^{23}$ unless future studies show their peculiar tendency towards 
an indolent behavior in PDGFRA-mutant syndrome. Lastly, genetic screening for PDGFRA should be performed in the relatives of patients affected by PDGFRA-mutant syndrome.

In conclusion, we report a kindred bearing a hitherto unreported dominantly inherited P653L PDGFRA mutation that contributes to the knowledge of the pathogenesis of PDGFRA-driven GISTs, and to correctly defining the disease formerly referred to as 'INF/NF3b' and later considered 'familial GIST'. As both these terms are misleading, we propose changing them to PDGFRA-mutant syndrome. In particular: (i) PDGFRA-mutant syndrome must be suspected whenever $\geq 2$ among inflammatory fibroid polyps (including fibrous tumors) and/or (gastric) GISTs are found in a single individual or in a family; gastrointestinal lipomas and/or large hands may be present along with these tumors; (ii) the diagnosis of PDGFRA-mutant syndrome requires the demonstration of a germline PDGFRA mutation; (iii) PDGFRAmutant syndrome patients should be investigated through endoscopy combined with endoscopic ultrasound and/or entero CT; (iv) oncological concern in PDGFRA-mutant syndrome appears to be mainly restricted to gastric tumors, as stomach proved to be selective for the PDGFRA mutations to progress to GIST, and GIST seems to be the only potentially malignant tumor in PDGFRA-mutant syndrome; (v) histological assessment of tumors is nevertheless recommended; (vi) surgery in PDGFRA-mutant syndrome tumors diagnosed as GIST is presently warranted according to current guidelines concerning GIST in general; (vii) surgery in patients affected by PDGFRA-mutant syndrome is also justified for non-GIST/non-gastric tumors, given the risk of developing bowel obstruction/intussusception; (viii) the relatives of individuals affected by PDGFRAmutant syndrome should be genetically screened to identify the affected mutant individuals.

\section{Acknowledgments}

We thank the family members for their cooperation. This work was supported by the Università Cattolica del Sacro Cuore (Linea D1 grants number 70200198 to RR, 70102040 to $\mathrm{MM}$, and 70201036 to LML).

\section{Disclosure/conflict of interest}

RR, GR and AC have received speakers' honoraria from Novartis and the remaining authors declare no conflict of interest.

\section{References}

1 Lipton S, Zuckerbrod M. Familial enteric neurofibromatosis. Med Times 1966;94:544-548.

2 Heimann R, Verhest A, Verschraegen J et al. Hereditary intestinal neurofibromatosis. I. A distinctive genetic disease. Neurofibromatosis 1988;1:26-32.
3 de Raedt T, Cools J, Debiec-Rychter M et al. Intestinal neurofibromatosis is a subtype of familial GIST and results from a dominant activating mutation in PDGFRA. Gastroenterology 2006;131:1907-1912.

4 Online Mendelian Inheritance in Man -OMIM- database number 606764, formerly independent with number 162220 http://www.omim.org/entry/606764? search = 606764 \&highlight $=606764$.

5 Corless CL, Barnett CM, Heinrich MC. Gastrointestinal stromal tumours: origin and molecular oncology. Nat Rev Cancer 2011;11:865-878.

6 Ricci R, Dei Tos AP, Rindi G. GISTogram: a graphic presentation of the growing GIST complexity. Virchows Arch 2013;463:481-487.

7 Schildhaus HU, Cavlar T, Binot E et al. Inflammatory fibroid polyps harbour mutations in the plateletderived growth factor receptor alpha (PDGFRA) gene. J Pathol 2008;216:176-182.

8 Lasota J, Wang ZF, Sobin LH et al. Gain-of-function PDGFRA mutations, earlier reported in gastrointestinal stromal tumors, are common in small intestinal inflammatory fibroid polyps. A study of 60 cases. Mod Pathol 2009;22:1049-1056.

9 Carney JA, Stratakis CA. Stromal, fibrous, and fatty gastrointestinal tumors in a patient with a PDGFRA gene mutation. Am J Surg Pathol 2008;32:1412-1420.

10 Chompret A, Kannengiesser C, Barrois $\mathrm{M}$ et al. PDGFRA germline mutation in a family with multiple cases of gastrointestinal stromal tumor. Gastroenterology 2004;126:318-321.

11 Spencer D. Recurrent familial inflammatory fibroid polyps of the small intestine. J Clin Pathol 1969;22:743.

12 Anthony PP, Morris DS, Vowles KD. Multiple and recurrent inflammatory fibroid polyps in three generations of a Devon family: a new syndrome. Gut 1984;25: 854-862.

13 Allibone RO, Nanson JK, Anthony PP. Multiple and recurrent inflammatory fibroid polyps in a Devon family ('Devon polyposis syndrome'): an update. Gut 1992;33:1004-1005.

14 Bayle S, Rossi P, Bagneres D et al. Ileum inflammatory fibroid polyp revealed by intussusception. About one familial case. Rev Med Interne 2005;26:233-237.

15 Pasini B, Matyakhina L, Bei T et al. Multiple gastrointestinal stromal and other tumors caused by plateletderived growth factor receptor alpha gene mutations: a case associated with a germline V561D defect. J Clin Endocrinol Metab 2007;92:3728-3732.

16 Ricci R, Arena V, Castri F et al. Role of p16/INK4a in gastrointestinal stromal tumor progression. Am J Clin Pathol 2004;122:35-43.

17 Yamamoto H, Oda Y, Kawaguchi K et al. c-kit and PDGFRA mutations in extragastrointestinal stromal tumor (gastrointestinal stromal tumor of the soft tissue). Am J Surg Pathol 2004;28:479-488.

18 Martini M, Santoro L, Familiari P et al. Inflammatory fibroid polyp of the gallbladder bearing a plateletderived growth factor receptor alpha mutation. Arch Pathol Lab Med 2013;137:721-724.

19 Sim NL, Kumar P, Hu J et al. SIFT web server: predicting effects of amino acid substitutions on proteins. Nucleic Acids Res 2012;40:W452-W457.

20 Adzhubei IA, Schmidt S, Peshkin L et al. A method and server for predicting damaging missense mutations. Nat Methods 2010;7:248-249.

21 Calabrese R, Capriotti E, Fariselli P et al. Functional annotations improve the predictive score of human 
disease-related mutations in proteins. Hum Mutat 2009;30:1237-1244.

22 Choi Y, Sims GE, Murphy S et al. Predicting the functional effect of amino acid substitutions and indels. PloS One 2012;7:e46688.

23 ESMO/European Sarcoma Network Working Group. Gastrointestinal stromal tumours: ESMO Clinical Practice Guidelines for diagnosis, treatment and follow-up. Ann Oncol 2014;25:iii21-iii26.

24 Joensuu H. Risk stratification of patients diagnosed with gastrointestinal stromal tumor. Hum Pathol 2008; 39:1411-1419.

25 Larghi A, Fuccio L, Chiarello G et al. Fine-needle tissue acquisition from subepithelial lesions using a forwardviewing linear echoendoscope. Endoscopy 2014;46: $39-45$.

26 Lasota J, Stachura J, Miettinen M. GISTs with PDGFRA exon 14 mutations represent subset of clinically favorable gastric tumors with epithelioid morphology. Lab Invest 2006;86:94-100.

27 Huss S, Wardelmann E, Goltz D et al. Activating PDGFRA mutations in inflammatory fibroid polyps occur in exons 12, 14 and 18 and are associated with tumour localization. Histopathology 2012;61:59-68.

28 COSMIC -catalogue of somatic mutations in cancerdatabase http://cancer.sanger.ac.uk/cancergenome/ projects/cosmic/.

29 Novelli M, Rossi S, Rodriguez-Justo M et al. DOG1 and CD117 are the antibodies of choice in the diagnosis of gastrointestinal stromal tumours. Histopathology 2010;57:259-270.

30 Daum O, Zalud R, Grossmann P et al. A case of imatinibnaive ileal fibrous stromal tumor with unusual morphology and double PDGFRA mutation. Appl Immunohistochem Mol Morphol 2010;18:484-485.

31 Daum O, Hatlova J, Mandys V et al. Comparison of morphological, immunohistochemical, and molecular genetic features of inflammatory fibroid polyps (Vanek's tumors). Virchows Arch 2010;456:491-497.

32 Liu TC, Lin MT, Montgomery EA et al. Inflammatory fibroid polyps of the gastrointestinal tract: spectrum of clinical, morphologic, and immunohistochemistry features. Am J Surg Pathol 2013;37:586-592.

$33 \mathrm{Kim}$ YI, Kim WH. Inflammatory fibroid polyps of gastrointestinal tract. Evolution of histologic patterns. Am J Clin Pathol 1988;89:721-727.

34 Kim MK, Higgins J, Cho EY et al. Expression of CD34, bcl-2, and kit in inflammatory fibroid polyps of the gastrointestinal tract. Appl Immunohistochem Mol Morphol 2000;8:147-153.

35 Daum O, Hes O, Vanecek T et al. Vanek's tumor (inflammatory fibroid polyp). Report of 18 cases and comparison with three cases of original Vanek's series. Ann Diagn Pathol 2003;7:337-347.

36 Antonescu CR, Viale A, Sarran L et al. Gene expression in gastrointestinal stromal tumors is distinguished by KIT genotype and anatomic site. Clin Cancer Res 2004;10:3282-3290.

37 Plesec TP. Gastrointestinal mesenchymal neoplasms other than gastrointestinal stromal tumors: focusing on their molecular aspects. Patholog Res Int 2011;2011: 952569 .

38 Agarwal R, Robson M. Inherited predisposition to gastrointestinal stromal tumor. Hematol Oncol Clin North Am 2009;23:1-13.

39 Al-Brahim N, Radhi J, Gately J. Synchronous epithelioid stromal tumour and lipoma in the stomach. Can J Gastroenterol 2003;17:374-375.

40 Liu X, Wilcox CM, Nodit L et al. Multiple gastrointestinal stromal tumors and lipomatosis. Arch Pathol Lab Med 2008;132:1825-1829.

41 Stolte M, Sticht T, Eidt S et al. Frequency, location, and age and sex distribution of various types of gastric polyp. Endoscopy 1994;26:659-665.

Supplementary Information accompanies the paper on Modern Pathology website (http://www.nature.com/ modpathol) 